morque électrique des lourds trains de marchandises. La proximité de chutes d'eau faciles à capter sera fréquemment une cause déterminante pour l'adoption de la traction électrique. Mais il semble qu'une extension importante de l'électrification des voies ferrćes doive trouver auprès des autorités militaires une opposition très vive, car la nécessité d'une liaison permanente entre un train électrique et l'usine génératrice constitue, en cas de mobilisation ou de campagne, un inconvénient extrêmement grave.

Rapport à la Société d'Encouragement pour l'Tndustrie nalionale (Bulletins de Mars et Mai rgi1), par M. de Varbreuze. - Voir La Houille Blanche de Scptembre et Octobre.

\section{INSTALLATIONS HYDRAULIQUES}

\section{BARRAGE D'EAST PARK}

Ce barrage a été construit par le Linited States Reclamation Service (analogue à notre Service des Amélioration Agricoles) pour assurer les irrigations de 6 ooo hectares de terraíns dans le comté d'Orland Glenn, en Californic. Il est établi à East Park, immédiatement à l'aval du confluent de I'Indian Creek et du Little Stony Crcek, soit à 5 kilomètres
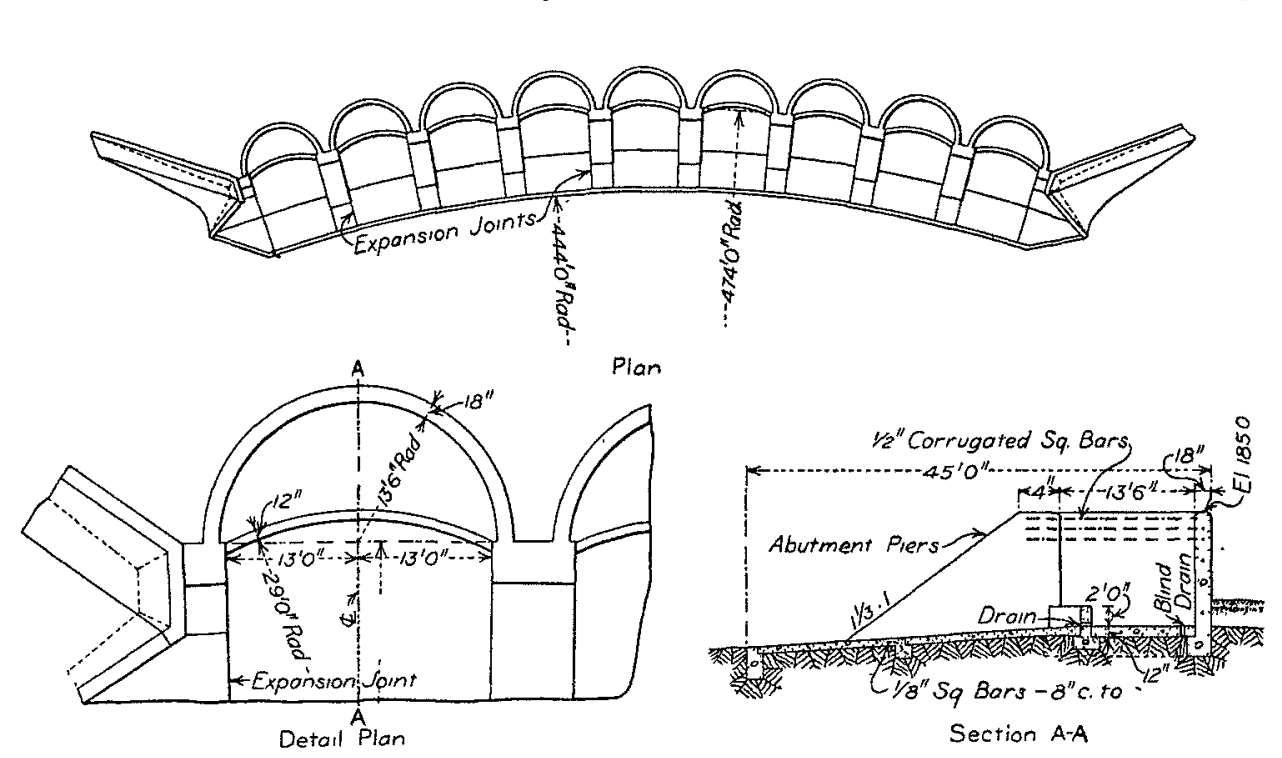

Fig. I. - Détails de construction du déversoir.
Le barrage d'East Park présente deux caractéristiques qui le distinguent un peu des autres onvages : son déversoir et ses joints de dilatation.

Barrage. - Le barrage esl établi au l'avers d'une gorge assez profonde et étroite, creusée dans un terrain formé de conglomérat assez résistant. Afin de s'assurer de la solidité de ce conglomérat, des sondages au diamant furent exéculés en divers points.

La hauteur du mur, compléc depuis le sommet du parapet (rgo p.), est de $27 \mathrm{~m} .43$ jusqu'au lit de la rivière (roo p.), el de $39 \mathrm{~m} .62$ jusqu'à la fondation ( $6 \circ \mathrm{p}$.).

Le profil est du type général triangulaire. L'épaisseur au sommet est de $3 \mathrm{~m}$. 05. Le parenent amont est vertical sur ro $\mathrm{m.67}$, puis est ensuile uniformément incliné de h pour roo sur la verticale. Le parement aval est d'abord constitué, au sommet, par un arc de cercle de $7 \mathrm{~m} .32$ de rayon, se continuant par une droile inclinće de 67 pour roo sur la verticale. La plus grande largeur à la base est de $26 \mathrm{~m}$. 2r.

Le mur est élabli cn plan suivant un are de cercle de $83 \mathrm{~m} .82$ de rayon. Sa longueur est de 76 mètres au sommet et l'angle au centre de l'are est do 5. $2^{\circ}$. Ce barrage présente donc une courbure assez inarquéc, insuffisante loutefois pour que cela augmente nolablemenl sa résistance, mais suffisante cependant pour créer une certainc compression dans le sens longitudinal, lorsque le réservoir esl plein.

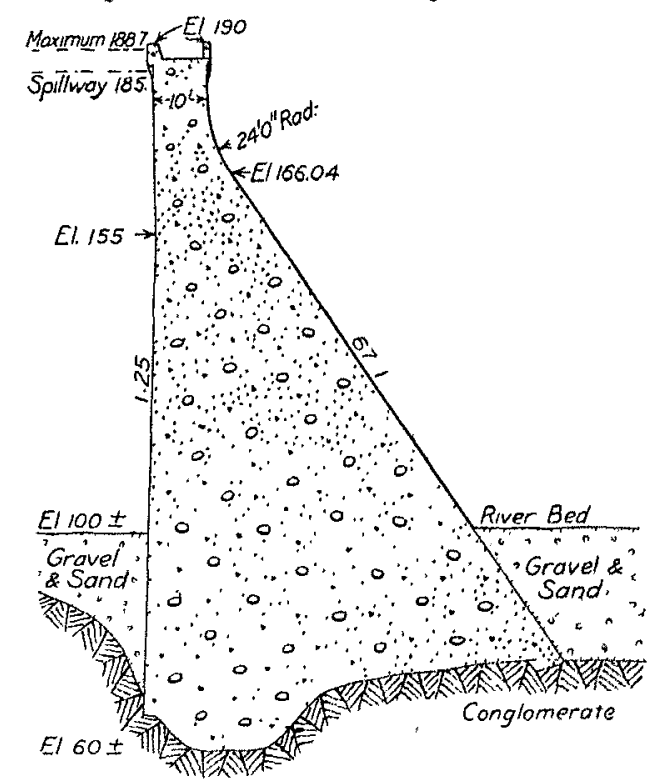

Fig. 2. - Profil du barrage. ¿i l'est de Stonyford, et à 5 kilomètres également en amont du conlluent du Lillle Stony Creek et du Stony Creek $\left({ }^{1}\right)$.

Les deux rivières ainsi barrées coulent dans les Coast Range Mountains, et leur bassin hydrologique d'alimentation est de 265 kilomètres carrés. La période des hautes eaux dure de décembre à juin. Les quantités d'eau écoulécs annuellement seraient, en millions de mètres cubes :

$\begin{array}{cccc}\text { de } & \text { II4 } & \text { I 70 } & 2 \times 2 \\ \text { en } & 1907 & \text { Igo8 } & \text { Igog }\end{array}$

Lce réservoir a une superficie de $77^{\circ}$ hectares el une capacité de 56,5 millions de mòtres cubes.

Pendanl la période de sécheresse, on ouvre les vannes de réglage, et l'eau retourne à la rivière, dont elle suit ainsi le cours pendant $52 \mathrm{~km}$. Elle est ensuite envoyée dans le canal d'irrigation, au moyen d'un petit barrage de dérivation.

(1) D'après l'Engineering Record du $\times 4$ juin rgrr.
A la cote roo, se trouve la conduite de vidange, de $1 \mathrm{~m} .52$ de diamètre, qui est commandée à l'amont au moyen de deux vannes disposées dans une lour de prise d'eau, en béton armé, accoléc au parement amont.

Les travaux furent adjugés le 5 octobro 1908 , mais les travaux proprement lits ne commencèrent qu'en mai Igog, par l'élablissement, à 220 mètres en amonl du barrage, d'un petit barrage dérivant l'eau dans un canal an bois qui la rejetait à 200 mètres à l'aval. Il avait d'ailleurs fallu mettre en état un chemin aboutissant a la plus prochaine slation de chemin de fer, distante de 20 kilomètres.

Les fouilles commencèrent en juin rgog, avec un texeavateur spécial, et furent continués avec cet appareil jusqu'à la cote 82 , où le congloméral commença à apparaître ; d'ail. leurs, en dessous de celle cole, la largeur de la fouille est réduite à peu de chose. Ia fondalion du mur a ćtó descendue partout jusqu'à ce que l'on put la faire reposer sur un conglomérat dur et résistant. 
Le mur de barrage est en béton, composé en moyenne de 2 partics de ciment pour 6 , I de sable, 5,5 de gravier fin, et 8,4 de gros gravier, correspondant à pen près au dosage 1 : $3: 7$. Etait considéré comme gros gravier ce qui passait à la grille de 3 pouces cl restait sur celle de I pouce $(25,40$ millimètres); comme gravicr fin, ce qui passait à la grille de $I$ pouce et restait sur celle de $1 / 4$ de pouce; enfin, le sable constituait ce qui passait à travers cette dernière grille. La densité moyenne de ce bélon a élé trouvée égale à 2,3 .

I.es essais de résistance sur des briqueltes, confectionnées avec le mortier employé, au dosage $\mathrm{I}: 3$, accusèrent une résistance à la traction de $\mathrm{i}, \mathrm{J}$ ? hilogrammes par centimètre carré, au bout de 7 jours, el de 17,67 kilogrammes au bout de 28 jours.

Des essais de perméabilité ayant montré que celle-ci était notablement augmentéc par l'adjonction de sable fin, on prépara un béton spécial pour les 3 premiers mètres du parement amont, en ajoutant, au sable ordinairement employé, 52 pour roo d'un sable plus fin extrait d'unc sablière voisine

L'entrepreneur établit son chantier de préparation du béton à 30 mètres à l'aval du barrage, à la cole r ro. Le sable et le gravier, extraits d'un ban voisin, ćlaient sélectionnés, comme il vient d'être dil, au moyen d'un cribleur rotatif, puis, après mesurage, élajent mélangés au ciment dans un malaxcur Ransome. A la sortie de co malaxeur, le béton étail vidé dans des wagonnels, et amené à un ćlévateur qui Je montait au niveau du chanticr de bétonnage. L'éncrgie nécessaire était produite par la vapeur, obtenue en brûlant les arbres du périmètre à submerger.

Pour les partics hautes du mur, construites après le i5 mai rgro, l'entrepreneur fut tenu, à causo de la haule température, de ne procéder au bétonnage que pendant la nuil, entre sept heures du soir et sept heures du matin, et à recouvrir son chanticr pendant le jour avec des claies humides.

L'ouvrage a été complètement terminé fin juillet rgro. La main-d'œuvre se composait de 38 hommes, la direction ct le contrôle de 4 personnes.

La dépense s'est élevée à 240000 dollars, dont to5000 pour le barrage proprement dit, i6 ooo pour le déversoir, 86 ooo pour l'acquisition du terrain et 33 ooo pour études et frais divers. Le prix de revicnt du mètre cubc ressort ainsi à 2,2 centimes environ.

DÉversorr. -- Ce déversoir n'est pas contigu au barrage, comme c'est généralement le cas, mais il est placé, à 400 mètres environ, au travers d'une petile dépression de terrain cntre le bassin du Tittle Stony Creek et celui d'un autre ruisseau.

Le maximum de débit des crues constatées a élé trouvé de 2.3o mètres cubes, mais le dévcrsoir a été prévu pour pouvoir évacuer 280 mètres cubes par seconde, sans que le niveau de l'eau dans le réservoir vienne à dépasser la crèle du parapêt du sommel du barrage. Afin d'avoir une grande longueur do la lame déversante, de manière à réduire la surélévation, on a constitué le déversoir par une série de 9 petites voûtes verticales, semi-circulaires, de $0 \mathrm{~m}$. I任 de diamètre extérieur, ce qui fait, pour la lame déversante une longueur de 120 mètres, alors que la dépression n'a que go mètres. Avec Ja formule Hazen el William, quii est dérivée de la formule Bazin, on a été ainsi amené à unc hauteur maxima de surélévalion de 3,7 pieds. Ia crète du déversoir a élé :rrasé à 5 pieds ( $\mathrm{m}$. 524) en dessous de la crête du parapet du barrage, soit à la cote $\mathrm{r} 85$.

Les détails de ce déversoir sont indiqués sur la figure ci-jointe. Les voûtes sont en bélon armé, de o m. 457 d'épaisseur, et 's'appuicnt sur des contreforts, également en béton armé, de $2 \mathrm{~m}$. 44 d'épaisseur. Pour amortir le choc de l'eau de la lame dévcrsante, on a créé une petite chambre de remous, au moyen d'unc murette, de om. 305 d'épaisscur et de o m. 6r de hauteur, établie à l'aval suivant un arc de cercle de $8 \mathrm{~m} .85$ de rayon.

Jonts de dilatation, - Les variations de température étant considérables dans la région où a été construit le barrage, et le réservoir pouvant être vide à l'entrée de l'hivcr, on a ménagé des joints verticaux de dilatation ou, plus exactement, on a provoqué des joinls de moindre résistance, où les fissures ne manqueront pas de so produire plutòt qu'ailleurs, pendant le retrait du bélon en hiver. Comme ces fissures sont prévurs à l'avance, qu'clles se produisent là où I'on a voulu qu'elles soient, et qu'elles doivent se refermer sous l'aclion de la compression longitudinale quand le réscrvoir est plein, cljes ne sont pas dangereuses.

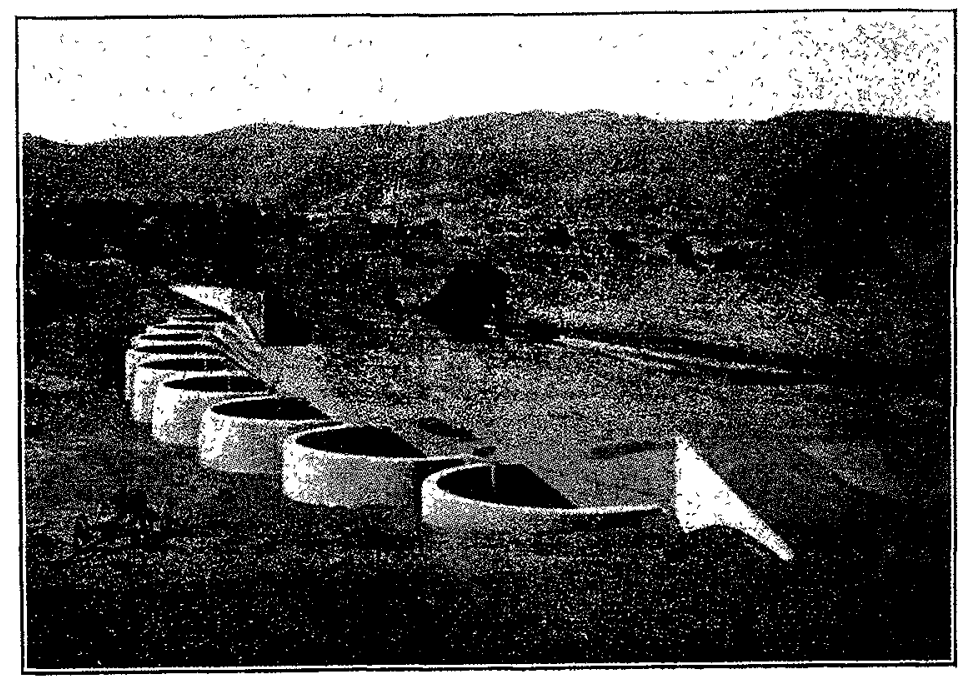

FIG. 3. - Vue dündéversoir d'East-Park.

Depuis la cote rgo jusqu'à la cote 155 , il y a un joint lous les $6 \mathrm{~m}$. 10 , et, de la cote 155 à la cote 125 , il y a un joint tous les $12 \mathrm{~m}$. 20. A chaque joint correspond un système de drains verticaux, aboutissant à une conduile de drainage débouchant à l'aval à la cote ion.

Le bétonnage fut cxécutć par blocs, correspondant à la largeur comprise entre deux joints, les formes transversales occupant la place des joints. Un couvre-joint, de $\mathrm{r}$ m. 22 de large sur o m. I5 d'ćpaisseur, taillé en biseau aux deux extrémilés, fut intercalé au travers des joints, à $\mathrm{I} m$. o5 du parement amont. Dès que la prise du béton fut faite, et les formes cnlevées, la surface du bélon des joints fut graissée, puis on coula ensuite du béton entre les deux blocs voisins, de manière à garnir le joint. Par suite du graissage, il se formait ainsi un joint de moindre résistance.

En été, les joints sont nalurellement fermés par suite de. la dilatation provoquée par la chaleur et de la compression de la voûte qui en résulte. Si le réscrvoir est en cau pendant l'hiver, la pression de l'eau tend à applatir la courbe du barrage, ce qui produil ancore une compression énergique des joints, el empêche l'ćconlement de l'eau. Enfin, si lo réscrvoir est vide pendant l'hiver, le retrait fait bicn ouvrir les joints, mais ceux-ci ne tardent pas à se refermer automatiquement dès que le réservoir vient à se remplir.

H. BELLEt. 